K Durairaj, J Mynett, M Dyke, A Marthandan. Neonatal Medicine, Norfolk and Norwich University Hospital, Norwich, UK

Background Vancomycin is commonly used in neonatal intensive care for suspected or proven Coagulase Negative Staphylococcus [CoNS] sepsis. Achieving the therapeutic level is important but subtherapeutic levels are common. There is little data to guide dosage adjustment.

Method A retrospective audit on vancomycin use was undertaken, using standard 24 hourly dose interval in extreme premature babies $[<29$ weeks]. Finding resulted in a change of dose frequency to 18hrly: a prospective audit was then performed.

Result Of the 27 extreme premature babies on 24 hrly vancomycin, $70 \%$ had sub-therapeutic levels; $26 \%$ had normal levels. 1 baby [4\%] with abnormal renal function had high level.

A subsequent prospective audit of 20 babies [dosed 18 hrly], showed $70 \%$ with sub-therapeutic levels, $25 \%$ with normal levels; one baby [5\%] with abnormal renal function had high level.

The commonest correction for sub-therapeutic levels was to increase the dose by $10 \%$; only $33 \%$ of repeat levels were then in normal range. Up to 4 dose increases were required to achieve the therapeutic target.

Conclusion Increasing vancomycin dosing frequency to 18 hrly produced no increase in the number of therapeutic or sub therapeutic levels, but might theoretically result in longer periods of therapeutic drug levels during the course.

On finding the sub-therapeutic levels, there should be flexibility in approach as $10 \%$ increase in dose is often ineffective; perhaps, change in frequency should also be considered. More studies are needed to guide the rapid achievement of therapeutic drug levels.

\section{PREMEDICATION FOR NEONATAL INTUBATION: CURRENT PRACTICE IN THE TERTIARY NEONATAL UNITS IN THE UNITED KINGDOM}

doi:10.1136/archdischild-2012-302724.1636

Y Singh, M Lester, V Ng, L Miall. Neonatal Medicine, The Leeds Teaching Hospitals NHS Trust, Leeds, UK

Background Evidence clearly shows that awake intubation is associated with a significantly higher intracranial pressure, higher blood pressure, and more variable heart rate than premedicated intubation. The last national survey was over 10 years ago. Recently there has been promising research on use of Propofol during neonatal intubation which showed it to be more effective than the morphine, atropine and suxamethonium.

Aims To establish and up to date census on the current use of premedications to facilitate neonatal intubation in the UK tertiary neonatal units.

Design and methods Telephone survey included all the 44 tertiary neonatal units in the UK. Professionals were asked about their current practice in use of pre-medications during neonatal intubation.

Results 44 tertiary neonatal units were contacted and all units use pre-medications to facilitate intubations. 40 of the 44 units (91\%) have written guideline or protocol. 6 premedication drugs are being used in 10 different combinations.

Combination of Fentanyl, Atropine and Suxamethonium is the most commonly used drug regimen used by 16 of 44 units $(36 \%)$ while $2^{\text {nd }}$ most popular regimen (used by $25 \%, 11$ of 44 units) included combination of Morphine, Atropine and Suxamethonium. Propofol is being used in only one unit.

Conclusion Use of premedications to facilitate intubation has become standard practice across the tertiary neonatal units in the UK. However practice varies in terms of choice, number and doses of premedication drugs. Six premedication drugs are being used in 10 different combinations/regimens which vary from 1-3 drugs.

\section{USE OF PREMEDICATION DRUGS FOR NEONATAL INTUBATION: IS THIS THE TIME TO THINK OF CHANGING CLINICAL PRACTICE?}

doi:10.1136/archdischild-2012-302724.1637

Y Singh, M Lester, V Ng, L Miall. Neonatal Medicine, The Leeds Teaching Hospitals NHS Trust, Leeds, UK

Background A recent national survey of tertiary neonatal units in the UK showed that six different premedication drugs are being used in ten different combinations. Preparation and administration of three premedication drugs, especially regimen having controlled drugs, may take significantly longer time and may delay intubation.

Aims and objectives:

To study the time taken for preparation and administration of commonest drug regimen (combination of Fentanyl, Atropine and Suxamethonium).

To study its efficacy during neonatal intubation.

Design and methods A prospective study in a tertiary neonatal setting in the UK included elective and semi-elective intubations. Neonatal intubations done in the delivery suite and emergency situation, where patient was collapsed, were excluded.

Results Data was collected from use of premedication drugs during 24 neonatal intubations. Mean time taken to obtain and prepare premedication drugs was 18 minutes (Range: 3-94 minutes) and mean time taken to administer premedication drugs was 3 minutes (Range: 1-10 minutes).

Mean time taken from insertion of laryngoscope in mouth to successful intubation was 5 minutes (Range: 1-24 min) and mean number of attempts were 2 (Range: $1-7$ attempts). Only $8 \%$ cases needed repeat premedication drugs.

Conclusion The average time taken for preparation and administration of three premedication drugs was 18 minutes which is significantly longer than expected for emergency situations. Use of single un-controlled premedication like Propofol can be quick and cost effective. Is this time to change our practice or do we need more randomised trials to study the efficacy of Propofol?

\section{WHAT IS THE EXTENT OF EXCIPIET INTAKE IN THE PICU PATIENT?}

doi:10.1136/archdischild-2012-302724.1638

${ }^{1}$ RE Isaac, ${ }^{2} \mathrm{C}$ Langley, ${ }^{2} \mathrm{~S}$ Sultana. 'Pharmacy Department/PICU, Birmingham Children's Hospital NHS Foundation Trust; ${ }^{2}$ Pharmacy School, Aston University, Birmingham, UK

Background Many medications used in PICU contain excipients which are classed as non-pharmaceutically active ingredients. There is increasing reports of adverse effects attributed to these agents. This study looks for recommendations of maximum levels and actual excipient intake in 5 PICU patients.

Methods EmBASe, Medline, UK and International guidelines were searched for adverse effects, pharmacokinetics, maximum doses for non-pharmaceutically active ingredients. The medication intake for 5 PICU patients was recorded and excipient content calculated where available.

Results There were 49 medications administered, no excipient information could be found for 8 . There was 235 excipients listed in the remaining 42 medicines, complete excipient information was available for $11 / 49$ medicines. Maximum upper limits were found for sorbitol $(0.5 \mathrm{~g} / \mathrm{kg})$, Propylene glycol $(25 \mathrm{mg} / \mathrm{kg})$ and hydroxybenzoates $(10 \mathrm{mg} / \mathrm{kg})$. Only a lethal dose was found for ethanol $(3.8 \mathrm{ml} /$ $\mathrm{kg}$ ), there was a recommendation that no ethanol should be contained in medicines for under 6 year olds.

Whilst excipient intake calculation was not complete due to lack of response or availability of information from the manufacturer, each patient received a medicine containing ethanol. Patient 3 and 
5 had above the sorbitol level $(0.9 \mathrm{~g}$ and $2.1 \mathrm{gram} / \mathrm{kg}$ respectively) Three patients received above the propylene glycol limit $(52.6 \mathrm{mg}$, $30 \mathrm{mg}$ and $190 \mathrm{mg} / \mathrm{kg}$ ), and at least one patient was getting $14.4 \mathrm{mg} /$ $\mathrm{kg}$ of hydroxybenzoates.

Conclusion When the information regarding quantities of excipients in medicines are available and calculated PICU patients are receiving significant amounts of excipients, some above the recommended safe limits.

\section{ADVERSE DRUG REACTIONS ASSOCIATED WITH CIPROFLOXACIN IN NEONATES}

doi:10.1136/archdischild-2012-302724.1639

${ }^{1} K A$ Kuruvilla, ${ }^{1} V$ James, ${ }^{2} \mathrm{M}$ Subramani, ${ }^{2 B}$ Mathew. 'Child Health \& Neonatology; ${ }^{2}$ Clinical Pharmacology, Christian Medical College \& Hospital Vellore, Vellore, India

Background Ciprofloxacin is used in many nurseries in developing countries. Data on drug concentrations and side effects of ciprofloxacin in neonates is limited.

Aims To study adverse drug reactions(ADR) associated with ciprofloxacin in term and preterm neonates and correlate them with drug levels.

Design Babies of 3 gestational age(GA) groups were enrolled: 37 (gp1), 32-36 (gp2) and 28-31 (gp3) weeks. Ciprofloxacin was administered twice daily at $10 \mathrm{mg} / \mathrm{kg} /$ dose IV. Lab parameters were done at baseline, day 3 and day 7, including peak and trough drug levels. Using Naranjo algorithm, babies who developed new symptoms after starting ciprofloxacin were classified into definite, probable, possible or doubtful ADR. Drug levels were correlated with ADR.

Results 165 babies receiving ciprofloxacin were enrolled. Predominant ADR were jaundice(79\%), rash(23\%), hyponatremia(28\%), anaemia(15\%) and hypokalemia(5\%). Using Naranjo algorithm, ProbableADR were cardiac arrhythmia, mucosal ulceration, renal failure and seizures. PossibleADR were rash, elevated liver enzymes, feed intolerance and leucopenia. ADRs were self-limited and treatable.

The mean (SD) trough and peak values of ciprofloxacin for the entire study population was $3.57(1.88)$ and 11.67 (3.66), respectively. Mean (SD) trough drug levels were gp 1-2.84 (1.54), gp 2-3.80 (1.9), gp 3-4.06 (1.98). GA did not seem to play a significant role in pharmacokinetics.

Drug levels higher than mean were seen in babies with rash, leucopenia and elevated LFT. Levels were adequate in babies with HIE, high in babies with NEC and lower in those with RDS.

Conclusions Ciprofloxacin can be considered safe for treating neonates.

\section{EFFICACY AND SAFETY OF MELATONIN IN NEONATES}

doi:10.1136/archdischild-2012-302724.1640

'S Aversa, 'L Marseglia, 'A Arco, 'G D'Angelo, 'E Cusumano, 'I Barberi, 'RJ Reiter, ${ }^{1} \mathrm{E}$ Gitto. 'Neonatal Intensive Care Unit, Department of Paediatrics, University of Messina, Messina, Italy; '2Department of Cellular and Structural Biology, The University of Texas Health Science Center, San Antonio, TX, USA

Background and Aims Newborns are more sensitive to oxidative stress than older infants. Melatonin, based on its properties as chronobiotic, antioxidant, or analgesic, offers perspectives of beneficial effects in neonatology. Aim of this study was to retrospectively review the efficacy and safety of melatonin administered at preterm and at term newborns in NICU.

Methods A retrospective patient record review of newborns treated with Melatonin in NICU of University of Messina (Italy) was performed.

Results 85 neonates were recruited and treated with Melatonin $(5-70 \mathrm{mg} / \mathrm{Kg} / \mathrm{die})$ in six previously published RCT. Melatonin has been given to 55 preterm infants with RDS and 30 at term newborns (10 with sepsis, 10 with perinatal asphyxia, and 10 with surgical abdominal malformations). That has always been given intravenously except for 10 septic newborns receiving oral administration. In our studies, melatonin treatment was able to reduce the level of proinflammatory cytokines, lipid peroxidation products and clinical parameters of inflammation and sepsis, and to improve the clinical outcome in terms of reduction of bronchodysplasia in preterm infants with RDS. None adverse event has been observed in our population of newborns treated with melatonin.

Conclusions To our knowledge, studies related to the toxicity of melatonin have not uncovered evidence of toxicity in humans even when given in very high doses. Our studies confirmed the potential role of melatonin as a treatment in different neonatal pathologies and the safety of its use in neonates at relatively high doses for short term and in various formulations.

\section{DIAZOXIDE OPENS THE CLOSING NEONATAL DUCTUS ARTERIOSUS}

doi:10.1136/archdischild-2012-302724.1641

K Momma. Pediatric Cardiology, Tokyo Women's Medical University, Tokyo, Japan

Background and Aims Sulfonylureas inhibit the ATP-sensitive potassium $\left(\mathrm{K}_{\mathrm{ATP}}\right)$ channel, are insulinogenic, and close the fetal ductus arteriosus. Diazoxide, a $\mathrm{K}_{\mathrm{ATP}}$ channel opener, is used for neonatal hyperinsulinemic hypoglycemia, and has been associated with the reopening of the ductus arteriosus. The aim of this study is to clarify ductus-opening effect of diazoxide.

Methods Neonatal rats were delivered by caesarian section nearterm and incubated at $34^{\circ} \mathrm{C}$. Diazoxide and pinacidil, another $\mathrm{K}_{\mathrm{ATP}}$ channel opener, were injected intraperitoneally immediately, or at one hour, or at four hours postnatally, and the ductus was studied 0.5 , and 1 hour later, with a rapid whole-body freezing method.

Results Diazoxide and pinacidil both induced hyperglycemia. Diazoxide and pinacidil delayed neonatal ductus closure following injection immediately after birth. At 2 hours, the control ductus was closed, whereas the ductus treated with $100 \mathrm{mg} / \mathrm{kg}$ of diazoxide at birth was widely patent with a diameter $40 \%$ of the fetal ductus. Ductus diameter at 60 minutes postnatally dilated from $10 \%$ to $40 \%$ with diazoxide. Diazoxide given to the closed ductus at 4 hours after birth did not open reopen it. The ductus was more sensitive to pinacidil than to diazoxide.

Conclusions Diazoxide and pinacidil open the closing ductus arteriosus of the neonatal rat. This study demonstrates that opening of $\mathrm{K}_{\mathrm{ATP}}$ channels results in opening of the ductus arteriosus, indicating that the $\mathrm{K}_{\text {ATP }}$ channel is physiologically and pharmacologically important in ductus opening. The ductus should be checked in the neonate before and after treatment with diazoxide.

\section{DESCRIBING THE USE OF OFF-LABEL AND NOT APPROVED MEDICATIONS IN A NEONATAL INTENSIVE CARE UNIT IN SOUTH BRAZIL}

doi:10.1136/archdischild-2012-302724.1642

${ }^{1} \mathrm{CG}$ Carvalho, ${ }^{2} \mathrm{M}$ Ribeiro, ${ }^{2} \mathrm{M}$ Bonilha, ${ }^{2} \mathrm{M}$ Fernandes Jr, ${ }^{2} \mathrm{RS}$ Procianoy, ${ }^{1} \mathrm{RC}$ Silveira. ${ }^{1}$ HCPA - UFRGS; ${ }^{2}$ UFRGS, Porto Alegre, Brazil

Background and Aims It is known that unlicensed medicines (unapproved) or used other way than directed in the label (off-label use) are widely prescribed in children. In the NICU, the severity of the patient justifies this type of prescription, evoking the risk-benefit ratio. We aimed to analyze the exposure to unapproved or off-label drugs in NICU in a tertiary university hospital in southern Brazil.

Method A descriptive cohort of drugs prescribed during hospitalization for 129 patients within 6 weeks. The drugs were classified as non-approved, and off-label for the dose, frequency, presentation, age or indication, according to FDA-approved e-lary. 